\title{
Exact exchange-correlation potential of effectively interacting Kohn-Sham systems
}

\author{
Shunsuke A. Sato $\odot^{1,2,{ }^{*}}$ and Angel Rubio $\circledast^{2,3,4}$ \\ ${ }^{1}$ Center for Computational Sciences, University of Tsukuba, Tsukuba 305-8577, Japan \\ ${ }^{2}$ Max Planck Institute for the Structure and Dynamics of Matter and Center for Free-Electron Laser Science, \\ Luruper Chaussee 149, 22761 Hamburg, Germany \\ ${ }^{3}$ Center for Computational Quantum Physics (CCQ), The Flatiron Institute, 162 Fifth Avenue, New York, New York 10010, USA \\ ${ }^{4}$ Nano-Bio Spectroscopy Group, Departamento de Fisica de Materiales, Universidad del País Vasco UPV/EHU, 20018 San Sebastián, Spain
}

(Received 29 June 2019; published 15 January 2020)

\begin{abstract}
Aiming to combine density functional theory (DFT) and wave-function theory, we study a mapping from the many-body interacting system to an effectively interacting Kohn-Sham system instead of a noninteracting Kohn-Sham system. Because a ground state of effectively interacting systems requires having a solution for the correlated many-body wave functions, this provides a natural framework to many-body wave-function theories such as the configuration interaction and the coupled-cluster method in the formal theoretical framework of DFT. Employing simple one-dimensional two-electron systems-namely, the one-dimensional helium atom, the hydrogen molecule, and the heteronuclear diatomic molecule-we investigate properties of many-body wave functions and exact exchange-correlation potentials of effectively interacting Kohn-Sham systems. As a result, we find that the asymptotic behavior of the exact exchange-correlation potential can be controlled by optimizing that of the effective interaction. Furthermore, the typical features of the exact noninteracting Kohn-Sham system, namely, a spiky feature and a step feature in the exchange-correlation potential for the molecular dissociation limit, can be suppressed by a proper choice of the effective interaction. These findings open a possibility to construct numerically robust and efficient exchange-correlation potentials and functionals based on the effectively interacting Kohn-Sham scheme.
\end{abstract}

DOI: 10.1103/PhysRevA.101.012510

\section{INTRODUCTION}

Density functional theory (DFT) is one of the most successful approaches to describe the ground-state properties of electronic systems [1,2]. A strong point of DFT is its computational feasibility and often it offers the best compromise of accuracy and computational costs. The feasible computational cost of DFT calculations can be achieved by the mapping from a fully interacting problem to a noninteracting problem based on the Hohenberg-Kohn theorems. In this mapping, all the complexities of the many-body problem are absorbed in the unknown exchange-correlation functional. Therefore, the accuracy of the DFT calculations essentially depends on the approximation of the exchange-correlation functionals. In the past decades, various exchange-correlation functionals have been developed to realize accurate descriptions of the electronic ground state such as local density approximations (LDA) [3,4], generalized gradient approximations (GGA) [5,6], meta-GGA [7-9], and hybrid functionals [10-12]. Furthermore, detailed studies have clarified several exact properties of the exact exchange-correlation functional and potential, such as asymptotic behavior of the potential in Coulombic systems $[5,13,14]$ and spiky features in molecular dissociation [15-19]. However, the systematic improvement of the exchange-correlation functionals and

\footnotetext{
*ssato@ccs.tsukuba.ac.jp
}

potentials is still a nontrivial task due to highly nonlinear and nonlocal natures of the density functional [20,21].

In contrast to DFT, wave-function theories [22] such as the configuration interaction and the coupled-cluster method offer a formal possibility to straightforwardly improve the accuracy up to the exact solution by increasing the size of the search space, although the required computational costs can easily become infeasible. Furthermore, in different fields, various methods have been developed for accurate description of the electronic structure, such as the $G W$ method [23-26] and the quantum Monte Carlo method [27-30]. However, such accurate approaches require huge computational costs and they become infeasible for large systems.

Because DFT and wave-function theory are based on different characteristics, the two approaches often have different points of strength and weakness. For example, DFT with conventional approximations tends to well capture the dynamical correlation effect [31], which requires many Slater determinants for accurate description of many-body wave functions, while DFT suffers from dramatical failures in describing the static correlation [17,32-34], which requires a few but more than one Slater determinant for accurate description of manybody wave functions. (The terms of static and dynamical correlation are further explained in Sec. IV A.) On the other hand, the wave-function theory can naturally capture the static correlation effect through the configuration interaction. Combining the two approaches, one may be able to realize an overall accurate theoretical description for quantum manybody systems. 
Several examples of combining DFT and wave-function theory exist in the literature. The hybrid functional $[10,11]$ is one of the successful examples, and it includes a part of nonlocal Fock-like exchange interaction based on wavefunction theory. Importantly, note that the hybrid functional is beyond the theoretical framework of conventional KohnSham DFT, but it is based on the generalized Kohn-Sham scheme [35], where interacting model systems are introduced to take into account a part of the electron-electron interaction but the systems are still represented by a single Slater determinant. Thus, the generalized Kohn-Sham systems are still described by fully uncorrelated wave functions. Another successful example is a combination of the configuration interaction method and DFT [36-41] where the Kohn-Sham orbitals based on DFT are used to construct the configuration interaction approach. Since the Kohn-Sham orbitals and their orbital energies may take into account a substantial amount of the dynamical correlation effect, the configuration interaction based on the DFT approach drastically improves the description.

In this work, we explore yet another possibility to accurately and efficiently combine DFT and wave-function theory, introducing a mapping between a fully interacting manybody system and an effectively interacting Kohn-Sham system instead of the noninteracting Kohn-Sham system. Fromager et al. have achieved the connection between a fully interacting system and a fictitious interacting system in terms of the range separation in multiconfigurational density functional theory [42]. Effectively interacting systems are not generally described by a single Slater determinant but require a correlated wave function. Therefore, wave-function theory may be naturally introduced within the formal theoretical framework of DFT. By optimally choosing the effective interaction, the electronic correlation may be efficiently described by the combination of DFT and wave-function theory. To explore more in detail the properties of an effectively interacting Kohn-Sham system, we investigate what the exact exchangecorrelation potential looks like for that correlated reference Kohn-Sham system, employing one-dimensional two-electron systems.

The paper is organized as follows. In Sec. II we first introduce a mapping between a fully interacting system and an effectively interacting Kohn-Sham system. Then, we describe the effective interactions that are used in this work. In Sec. III, numerical methods to compute exact exchangecorrelation potentials of effectively interacting Kohn-Sham systems are described. In Sec. IV, we investigate exact exchange-correlation potentials and properties of effectively interacting Kohn-Sham systems for one-dimensional helium atoms, hydrogen molecules, and heteronuclear diatomic molecules. Finally, our findings are summarized in Sec. V.

\section{METHODS}

We introduce a mapping from a fully interacting manybody system to an effectively interacting Kohn-Sham system. For this purpose, we first consider a fully interacting $\mathrm{N}$ electron system. The ground state of the electronic system is described by the following Schrödinger equation:

$$
\hat{H} \Psi\left(\boldsymbol{r}_{1}, \ldots, \boldsymbol{r}_{N}\right)=E_{\mathrm{gs}} \Psi\left(\boldsymbol{r}_{1}, \ldots, \boldsymbol{r}_{N}\right),
$$

with the nonrelativistic many-body Hamiltonian given by (atomic units are used unless stated otherwise)

$$
\hat{H}=\sum_{i=1}^{N}\left[-\frac{1}{2} \nabla_{i}^{2}+v_{\mathrm{ext}}\left(\boldsymbol{r}_{i}\right)\right]+\frac{1}{2} \sum_{i \neq j} W\left(\boldsymbol{r}_{i}-\boldsymbol{r}_{j}\right),
$$

where $v_{\text {ext }}(\boldsymbol{r})$ is a one-body external potential and $W(\boldsymbol{r})$ is the electron-electron interaction.

Then, we introduce an effectively interacting Kohn-Sham system that satisfies the following interacting Kohn-Sham equation:

$$
\hat{H}_{\mathrm{KS}} \Phi_{\mathrm{KS}}\left(\boldsymbol{r}_{1}, \ldots, \boldsymbol{r}_{N}\right)=E_{\mathrm{KS}} \Phi_{\mathrm{KS}}\left(\boldsymbol{r}_{1}, \ldots, \boldsymbol{r}_{N}\right),
$$

with the interacting Kohn-Sham Hamiltonian

$$
\hat{H}_{\mathrm{KS}}=\sum_{i=1}^{N}\left[-\frac{1}{2} \nabla_{i}^{2}+v_{\mathrm{KS}}\left(\boldsymbol{r}_{i}\right)\right]+\frac{1}{2} \sum_{i \neq j} W_{\mathrm{eff}}\left(\boldsymbol{r}_{i}-\boldsymbol{r}_{j}\right),
$$

where $v_{\mathrm{KS}}(\boldsymbol{r})$ is the one-body Kohn-Sham potential and $W_{\text {eff }}(\boldsymbol{r})$ is an arbitrary effective interaction. Here, the KohnSham potential $v_{\mathrm{KS}}(\boldsymbol{r})$ is introduced such that the ground-state density of the fully interacting system is reproduced by that of the Kohn-Sham system:

$$
\begin{aligned}
\rho(\boldsymbol{r}) & =N \int d \boldsymbol{r}_{2} \cdots d \boldsymbol{r}_{N}\left|\Psi\left(\boldsymbol{r}, \boldsymbol{r}_{2}, \ldots, \boldsymbol{r}_{N}\right)\right|^{2} \\
& =N \int d \boldsymbol{r}_{2} \cdots d \boldsymbol{r}_{N}\left|\Phi_{K S}\left(\boldsymbol{r}, \boldsymbol{r}_{2}, \ldots, \boldsymbol{r}_{N}\right)\right|^{2} .
\end{aligned}
$$

Note that here the Kohn-Sham potential $v_{\mathrm{KS}}(\boldsymbol{r})$ is not uniquely constructed since a constant shift of the potential does not affect the ground-state density. Furthermore, the reconstruction of the corresponding energy functional or exchangecorrelation functional is not trivial. Levy and Zahariev proposed a simple way to evaluate the exact interacting groundstate energy as a sum of orbital energies based on the arbitrary constant term in the Kohn-Sham potential [43]. In this work, we extend this idea to interacting Kohn-Sham systems and set the arbitrary constant in the Kohn-Sham potential $v_{\mathrm{KS}}(\boldsymbol{r})$ such that the ground-state energy of the Kohn-Sham system $E_{\mathrm{KS}}$ is identical to that of the fully interacting system $E_{\mathrm{gs}}$.

The Hohenberg-Kohn theorems offer one-to-one correspondence between the ground-state density $\rho(\boldsymbol{r})$ and the onebody external potential $v_{\text {ext }}(\boldsymbol{r})$ once the interaction $W(\boldsymbol{r})$ is given [1]. Because the Hohenberg-Kohn theorems are not limited to the Coulomb interaction but are generally applicable to arbitrary interactions, there is one-to-one correspondence between the one-body potential $v_{\mathrm{KS}}(\boldsymbol{r})$ and the corresponding ground-state electron density once the effective interaction $W_{\text {eff }}(\boldsymbol{r})$ is defined. Thus, once both interactions, $W(\boldsymbol{r})$ and $W_{\text {eff }}(\boldsymbol{r})$, are given, there is a one-to-one correspondence between $v_{\mathrm{ext}}(\boldsymbol{r})$ and $v_{\mathrm{KS}}(\boldsymbol{r})$ through the common ground-state density $\rho(\boldsymbol{r})$, resulting in a one-to-one correspondence between the fully interacting many-body system and the effectively interacting Kohn-Sham system. If the effective interaction is set to zero, $W_{\text {eff }}(\boldsymbol{r})=0$, this one-to-one mapping 
is reduced to the conventional Kohn-Sham mapping between the interacting system and the corresponding noninteracting Kohn-Sham system.

For later convenience, we decompose the Kohn-Sham potential $v_{\mathrm{KS}}(\boldsymbol{r})$ into the external potential $v_{\mathrm{ext}}(\boldsymbol{r})$, the residual Hartree potential $v_{\mathrm{R}-\mathrm{H}}(\boldsymbol{r})$, and the exchange-correlation potential $v_{\text {xc }}(\boldsymbol{r})$. Here, we define the residual Hartree potential as

$$
v_{\mathrm{R}-\mathrm{H}}(\boldsymbol{r})=\int d \boldsymbol{r}^{\prime} \Delta W_{\mathrm{res}}\left(\boldsymbol{r}-\boldsymbol{r}^{\prime}\right) \rho\left(\boldsymbol{r}^{\prime}\right),
$$

where $\Delta W_{\text {res }}(\boldsymbol{r})$ is the residual interaction defined as $\Delta W_{\text {res }}(\boldsymbol{r})=W(\boldsymbol{r})-W_{\text {eff }}(\boldsymbol{r})$. Note that if the effective interaction is set to zero, $W_{\text {eff }}(\boldsymbol{r})=0$, the residual Hartree potential $v_{\mathrm{R}-\mathrm{H}}(\boldsymbol{r})$ is reduced to the conventional Hartree potential, $v_{H}(\boldsymbol{r})=\int d \boldsymbol{r}^{\prime} W\left(\boldsymbol{r}-\boldsymbol{r}^{\prime}\right) \rho\left(\boldsymbol{r}^{\prime}\right)$. Furthermore, if $W_{\mathrm{eff}}(\boldsymbol{r})=W(\boldsymbol{r})$, the residual Hartree potential vanishes. Therefore, the residual Hartree potential, Eq. (6), can be seen as a natural extension of the conventional Hartree potential.

Then, we define the exchange-correlation potential as the rest of the Kohn-Sham potential:

$$
v_{\mathrm{xc}}(\boldsymbol{r}):=v_{\mathrm{KS}}(\boldsymbol{r})-v_{\mathrm{ext}}(\boldsymbol{r})-v_{\mathrm{R}-\mathrm{H}}(\boldsymbol{r}) .
$$

The exchange-correlation potential in Eq. (7) is reduced to the conventional exchange-correlation potential of the noninteracting Kohn-Sham system if the effective interaction $W_{\text {eff }}(\boldsymbol{r})$ is set to zero. Thus, Eq. (7) can be also seen as an extension of the conventional exchange-correlation potential.

In this work, we investigate effectively interacting KohnSham systems and their exact exchange-correlation potentials to explore a possibility to combine DFT and wavefunction theory. To practically elucidate the effectively interacting Kohn-Sham systems, we consider the example of one-dimensional spin-1/2 two-electron systems. As the electron-electron interaction, we employ the one-dimensional soft Coulomb potential

$$
W(x)=\frac{1}{\sqrt{x^{2}+\sigma^{2}}},
$$

where $\sigma$ is a softening parameter, which is set to 0.5 a.u.

As reference (interacting and noninteracting) Kohn-Sham systems, we consider three kinds of systems in this work. The first one is a noninteracting Kohn-Sham system, where the effective interaction $W_{\text {eff }}(x)$ is set to zero. Note that this choice is nothing but the conventional noninteracting KohnSham system or standard DFT [2]. The second one is a 1/4interacting system, where the effective interaction is set to the quarter of the bare soft Coulomb interaction, $W_{\mathrm{eff}}(x)=$ $W(x) / 4$. The third one is a long-range interacting system, where $W_{\text {eff }}(x)=\operatorname{erf}\left(\sqrt{x^{2}+\sigma^{2}} / a_{0}\right) W(x)$ with the Bohr radius $a_{0}$. Thus, the short-range part of the Coulomb interaction is ignored in the long-range interacting system.

\section{NUMERICAL DETAILS}

Here, we describe numerical procedures to compute the exact exchange-correlation potentials of the Kohn-Sham systems. For the noninteracting two-particle Kohn-Sham system, one can easily compute the exact Kohn-Sham potential as

$$
v_{\mathrm{KS}}(x)=\frac{1}{2} \frac{1}{\sqrt{\rho(x)}} \frac{\partial^{2}}{\partial x^{2}} \sqrt{\rho(x)}+E_{\mathrm{gs}} .
$$

For interacting Kohn-Sham systems, we employ the following iterative scheme to obtain the exact Kohn-Sham potential that reproduces the target ground-state density $\rho^{\text {target }}(x)$.

(i) Start from an initial guess of the Kohn-Sham potential, $v_{\mathrm{KS}}^{(i=0)}(x)$. In this work, we employ the external potential $v_{\text {ext }}(x)$ as the initial guess.

(ii) Compute the ground-state density $\rho^{(i)}(x)$, solving the interacting Kohn-Sham equation, Eq. (3), with the trial potential $v_{\mathrm{KS}}^{(i)}(x)$. by

(iii) Then, evaluate the deviation from the target density

$$
r_{\mathrm{error}}^{(i)}=\int d x\left|\rho^{(i)}(x)-\rho^{\mathrm{target}}(x)\right|^{2} .
$$

(iv) If the error $r_{\text {error }}^{(i)}$ is larger than a given threshold $\eta$, the trial Kohn-Sham potential is updated by the following formula:

$$
v_{\mathrm{KS}}^{(i+1)}(x)=v_{\mathrm{KS}}^{(i)}(x)+\alpha_{i} \frac{\rho^{(i)}(x)-\rho^{\operatorname{target}}(x)}{\rho^{(i)}(x)+\rho^{\operatorname{target}}(x)+\epsilon},
$$

where $\alpha_{i}$ is a mixing parameter and $\epsilon$ is a small positive number. In this work, we set $\epsilon$ to $10^{-8}$ a.u. and choose the mixing parameter $\alpha_{i}$ such that the error of the updated density $r_{\text {error }}^{(i+1)}$ in the next iteration becomes smaller than that of the previous iteration $r_{\text {error }}^{(i)}$. In practical calculations, we set the initial value of $\alpha_{0}=0.1$. In the iterative procedure, we employ an acceptance-rejection procedure to determine the value of $\alpha_{i}$. At each iteration, the initial guess of $\alpha_{i}$ is evaluated as $\alpha_{i}=1.1 \alpha_{i-1}$. If the computed error $r_{\text {error }}^{(i+1)}$ is smaller than the previous error $r_{\text {error }}^{(i)}$, the guess value of $\alpha_{i}$ is accepted. If the computed error is not smaller than the previous error, the guess value of $\alpha_{i}$ is rejected, and the new guess value is set as half of the previous guess value. This procedure is recursively repeated until $r_{\text {error }}^{(i+1)}$ becomes smaller than $r_{\text {error }}^{(i)}$

(v) Repeat the above iterative procedure until the error $r_{\text {error }}^{(i)}$ becomes smaller than a given threshold $\eta$. In this work, we set the threshold $\eta$ to $10^{-9}$ a.u.

Note that similar iterative approaches to compute the exact Kohn-Sham potential have been proposed $[44,45]$. However, any stable approaches can be employed in this work because the size of the problems is small, and the accurate results can be obtained with reasonable computational costs.

\section{RESULTS}

\section{A. 1D helium atom}

First, we investigate the effectively interacting Kohn-Sham systems of the one-dimensional helium atom. To describe the helium atom, we employ the following external potential:

$$
v_{\mathrm{ext}}^{\mathrm{He}}(x)=-\frac{2}{\sqrt{x^{2}+\sigma^{2}}} .
$$

Figure 1(a) shows the exact ground-state density of the helium atom, obtained by numerically solving the two-dimensional Schrödinger equation with the conjugate gradient method. 


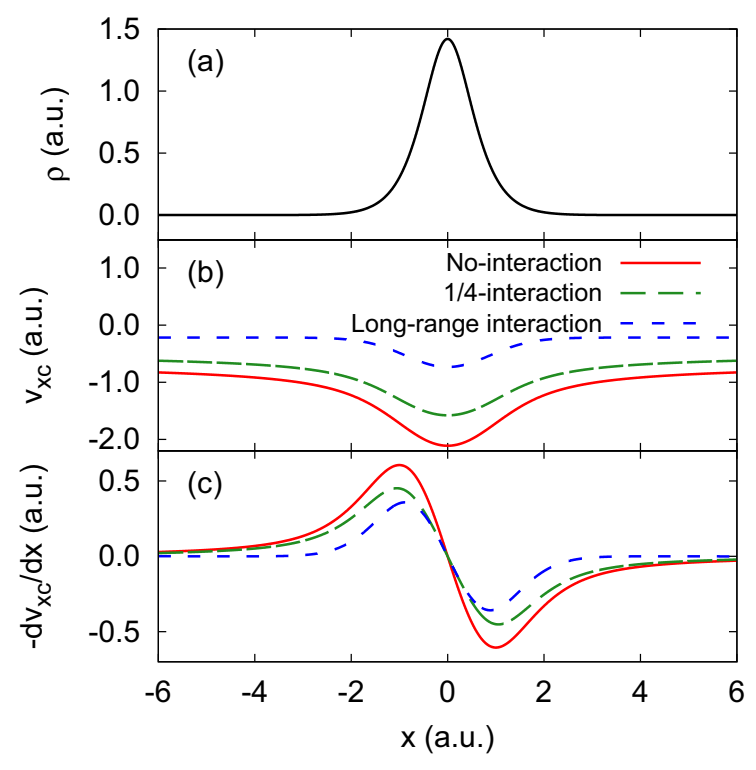

FIG. 1. (a) The exact ground-state density $\rho(x)$ of the onedimensional helium atom. (b) The exact exchange-correlation potentials for the effectively interacting Kohn-Sham systems: the red solid line shows the results for the noninteracting system, $W_{\text {eff }}(x)=0$; the green dashed line shows the results for the 1/4-interacting system, $W_{\text {eff }}(x)=1 /\left(4 \sqrt{x^{2}+\sigma^{2}}\right)$; and the blue dotted line shows the longrange interacting system, $W_{\text {eff }}(x)=\operatorname{erf}\left(\sqrt{x^{2}+\sigma^{2}} / a_{0}\right) / \sqrt{x^{2}+\sigma^{2}}$. (c) The force field $-d v_{\mathrm{xc}}(x) / d x$ of each exchange-correlation potential in panel (b).

Figure 1(b) shows the exact exchange-correlation potentials with respect to the three different interacting Kohn-Sham systems. The different effective interactions provide substantially different exchange-correlation potentials. One may see that the long-range tail of the exact exchange-correlation potential of the long-range interacting system (blue dashed line) decays faster than those of the noninteracting and the 1/4-interacting Kohn-Sham systems. To clearly compare the exchange-correlation potentials of the different Kohn-Sham systems, Figure 1(c) shows the exchange-correlation force field, $-d v_{\mathrm{xc}}(x) / d x$. One can clearly see that the exchangecorrelation force field of the long-range interacting KohnSham system shows much faster decay than the other systems. Furthermore, the long-range interacting system has the smallest force field over the whole spatial region. These features indicate a possibility to control the asymptotic behaviors of the exchange-correlation potential by choosing a suitable effective interaction used to set the reference interacting KohnSham system.

Then, we investigate details of the asymptotic behavior of the exchange-correlation potentials. Figure 2 shows the long-range behavior of the exact exchange-correlation potentials shown in Fig. 1(b). In Fig. 2(a), $v_{x c}(x)$ of the noninteracting system is shown as the red line, while an analytic curve, $-1 / x+c$ with $c=-0.661$ a.u., is described by the red circles. Thus, $v_{\mathrm{xc}}(x)$ of the noninteracting system has $-1 / x$ asymptotics. This behavior is known as the asymptotic behavior of the exchange-correlation potential of a Coulombic system [5]. In Fig. 2(b), $v_{\mathrm{xc}}(x)$ of the 1/4-interacting system is shown as the green line, while the analytic curve,

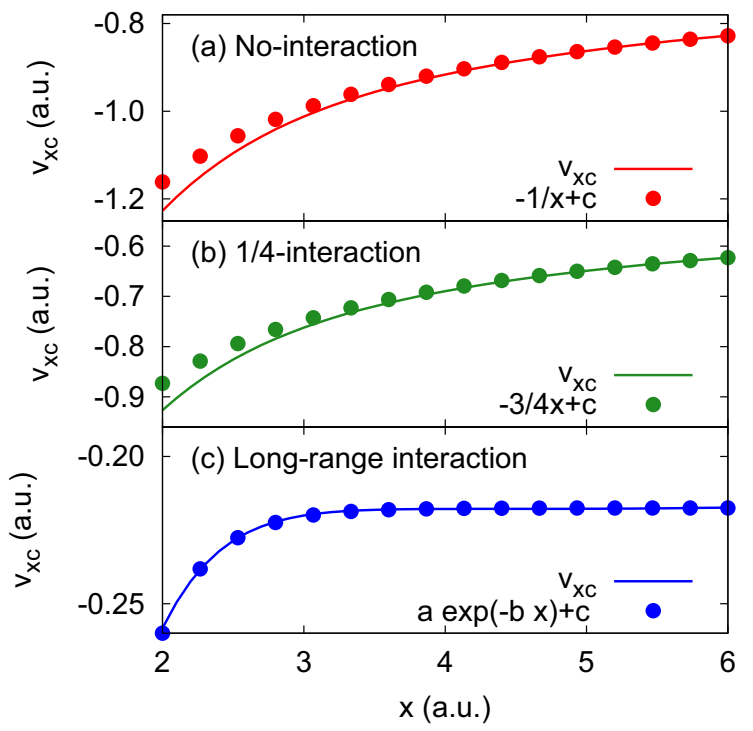

FIG. 2. Asymptotic behaviors of the exact exchange-correlation potentials for (a) the noninteracting, (b) the 1/4-interacting, and (c) the long-range-interacting Kohn-Sham systems.

$-\frac{3}{4 x}+c$ with $c=-0.498$ a.u., is described by the green circles. Therefore, $v_{\mathrm{xc}}(x)$ of the $1 / 4$-interacting system has $-\frac{3}{4 x}$ asymptotics, which is different from the conventional asymptotics of the exchange-correlation potential. The $-\frac{3}{4 x}$ asymptotics corresponds to the asymptotic behavior of the residual interaction of the 1/4-interacting system, $\Delta W_{\text {res }}(x)=$ $W(x)-W_{\text {eff }}(x)=3 /\left(4 \sqrt{x^{2}+\sigma^{2}}\right)$. Therefore, the asymptotic behavior of $v_{\mathrm{xc}}(x)$ of an effectively interacting Kohn-Sham system can be characterized by that of the residual interaction. In Fig. 2(c), $v_{\mathrm{xc}}(x)$ of the long-range-interacting system is shown as the blue line, while the analytical curve, $a \exp [-b x]+c$, is described by the blue circles. Here, the parameters are set as follows: $a=-9.41$ a.u., $b=2.7$ a.u., and $c=-0.2175$ a.u.

One sees that the asymptotic decay of $v_{\mathrm{xc}}$ is slower than that of the residual interaction, $\Delta W_{\text {res }}(x)=W(x)-W_{\text {eff }}(x)=$ $\operatorname{erfc}\left(\sqrt{x^{2}+\sigma^{2}} / a_{0}\right) / \sqrt{x^{2}+\sigma^{2}}$. This fact can be understood by the asymptotic behavior of the electron density: The residual interaction decays so fast that the asymptotics of $v_{\mathrm{xc}}(x)$ is dominated by that of the electron density in order to correctly remove the self-interaction error due to the local density. This feature may indicate that the local density approximation for the exchange-correlation functional may work well for the long-range interacting Kohn-Sham system because the exchange-correlation potential vanishes once the density vanishes and the nonlocality in the exchange-correlation potential is expected to be milder than that of the noninteracting KohnSham system.

Due to effective interactions $W_{\text {eff }}(x)$, the ground-state wave function of interacting Kohn-Sham Hamiltonian in Eq. (3) is not generally described by a single Slater determinant, but it requires multi-Slater determinants for the accurate description. Therefore, in the interacting Kohn-Sham system, the electronic correlation can be treated separately: A part of the correlation can be treated as the explicit correlation in the many-body Kohn-Sham wave function, while the other 


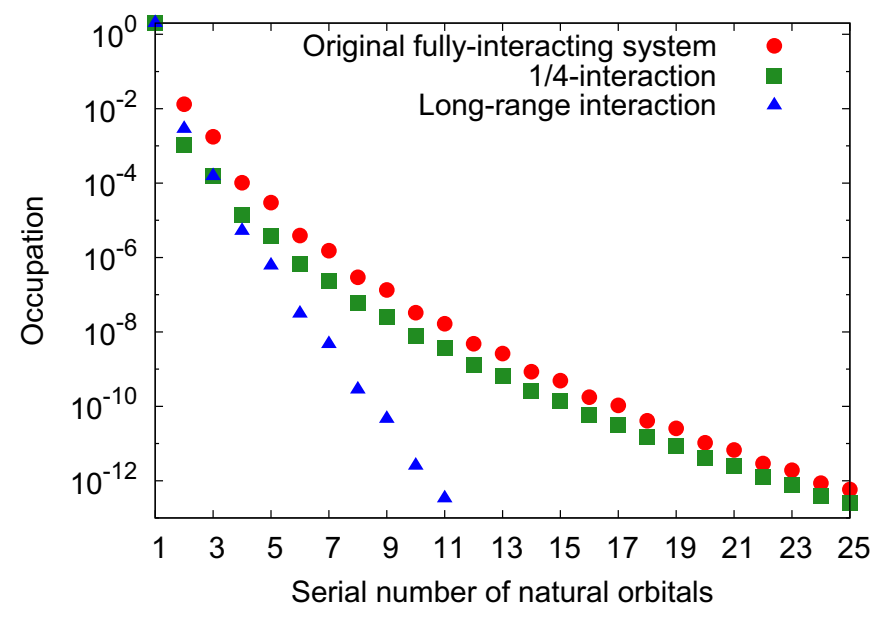

FIG. 3. Distribution of natural occupation over a few tens of natural orbitals. The results of the fully interacting system (red circle), the 1/4-interacting system (green square), and the long-range interacting system (blue triangle) are shown.

part can be implicitly treated in the exchange-correlation potential, $v_{\mathrm{xc}}(x)$, or the corresponding density functional $E_{\mathrm{xc}}[\rho(x)]$. This separation enables one to combine DFT and wave-function theory in order to efficiently describe the electronic correlation. For example, the static correlation may be efficiently treated by the multiconfiguration interaction as the explicit correlation in the correlated wave function, while the dynamical correlation may be treated by DFT through the exchange-correlation functional. To explore such a possibility, we next investigate the explicit correlation in the effectively interacting Kohn-Sham wave functions. For this purpose, we consider the eigendecomposition of the one-body reduced density matrix

$$
\begin{aligned}
\rho_{1 \mathrm{RDM}}\left(x, x^{\prime}\right) & =2 \int d x_{2} \Psi_{\mathrm{KS}}\left(x, x_{2}\right) \Psi_{\mathrm{KS}}^{*}\left(x^{\prime}, x_{2}\right) \\
& =\sum_{i=1} n_{i} \phi_{i}(x) \phi_{i}^{*}\left(x^{\prime}\right)
\end{aligned}
$$

where eigenvectors $\phi_{i}(x)$ are known as natural orbitals [46] and eigenvalues $n_{i}$ are seen as their occupations. Here, we assume that the occupation numbers are arranged in decreasing order, $n_{i} \geqslant n_{i+1}$. Since we treat the spatial part of the spin-singlet wave function $\Psi_{\mathrm{KS}}\left(x, x^{\prime}\right)$, natural occupations are restricted as $0 \leqslant n_{i} \leqslant 2$.

The occupation distribution deeply links to the number of configurations that is required to accurately describe a correlated electronic wave function $[22,46]$. If only a small number of orbitals have substantial occupations and the others have negligible occupations, the correlated system can be described by a small number of Slater determinants. In contrast, if a larger number of orbitals have substantial occupations, a larger number of configurations are required. Electronic correlation in the first case is called static correlation, while that in the latter case is called dynamical correlation.

Figure 3 shows the distribution of the occupations $n_{i}$ of the correlated ground-state wave function of the one-dimensional helium atom. The red circles show the occupation distribution of the fully interacting system, the green squares show that of the 1/4-interacting Kohn-Sham system, and the blue triangles show that of the long-range interacting Kohn-Sham system. As seen from the figure, the occupations of the higher natural orbitals are significantly suppressed in the effectively-interacting Kohn-Sham systems, compared with the fully interacting problem. Thus, a large part of the electronic correlation is transferred from the explicit correlation in the many-body wave function to the exchange-correlation functional. Furthermore, in Fig. 3, the long-range interacting system shows the rapid decrease of the occupations of the higher natural orbitals. This fact indicates that, in the long-range interacting Kohn-Sham system, the significant part of the dynamical correlation is transferred to the exchangecorrelation functional, while the static correlation is treated as the explicit correlation in the reference correlated wave function. Importantly, we note that the long-range interacting Kohn-Sham system has the weakest exchange-correlation potential with the fastest asymptotic decay among all the investigated Kohn-Sham systems (see Fig. 1). Therefore, this fact clearly demonstrates that a proper choice of the effective interaction enables one to efficiently decompose the electronic correlation into the exchange-correlation functional part and the explicit wave-function correlation part, resulting in an efficient description of the electronic correlation based on a combination of DFT and wave-function theory.

Note that the results presented in this section do not significantly depend on the choice of the softening parameter $\sigma$. The exact exchange-correlation potentials for $\sigma=1$ a.u. bear no qualitative difference to those presented in Fig. 1.

\section{B. 1D $\mathrm{H}_{2}$ molecule}

Next, we investigate the effectively interacting Kohn-Sham systems of the one-dimensional hydrogen molecule. To describe the hydrogen molecule, we employ the following external potential:

$$
v_{\mathrm{ext}}^{\mathrm{H}_{2}}(x)=-\frac{1}{\sqrt{\left(x-\frac{R}{2}\right)^{2}+\sigma^{2}}}-\frac{1}{\sqrt{\left(x+\frac{R}{2}\right)^{2}+\sigma^{2}}},
$$

where $R$ is the distance of the hydrogen atoms. In this work, we set $R$ to 5 a.u.

Figure 4(a) shows the exact ground-state electron density of the one-dimensional hydrogen molecule, obtained by numerically solving the two-dimensional Schrödinger equation with the conjugate gradient method. At the center of the two hydrogen atoms, the electron density becomes close to zero. Figure 4(b) shows the exact exchange-correlation potentials of the noninteracting and the effectively interacting Kohn-Sham systems. The exact exchange-correlation potential of the noninteracting system (red solid line) shows a spiky structure at the center. This spiky structure was investigated in the previous works for the strongly correlated system $[17,18,47,48]$. One sees that the peak structure is strongly suppressed in the 1/4-interacting Kohn-Sham system (green dashed line). Furthermore, the exchange-correlation potential of the long-range interacting Kohn-Sham system (blue dotted line) shows a very smooth feature around the central region. This fact indicates that while the strong correlation effect is encoded in the spiky structure in the noninteracting Kohn-Sham system, that 


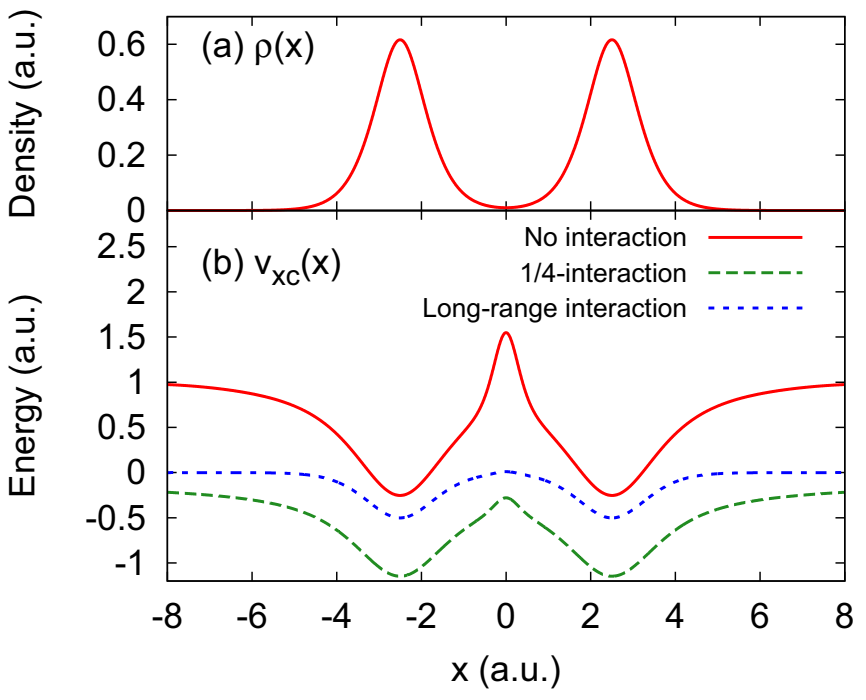

FIG. 4. (a) The exact ground-state density $\rho(x)$ of the onedimensional hydrogen molecule. (b) The exact exchange-correlation potentials for the effectively interacting Kohn-Sham systems. The results for the noninteracting system (red solid line), the 1/4interacting system (green dashed line), and the long-range interacting system (blue dotted line) are shown.

of the effectively interacting Kohn-Sham systems is directly taken care of through the explicit correlation of the reference wave function. Therefore, by properly choosing the effective interaction of the Kohn-Sham system, one can transfer the electronic correlation effect from the exchange-correlation functional or potential to the explicit correlation in the wave function in order to reduce the complexity for developing accurate approximations for the remaining exchange-correlation functionals and potentials.

\section{1D heteronuclear diatomic molecule}

Finally, we investigate the effectively interacting KohnSham systems of the one-dimensional heteronuclear diatomic molecule. In order to describe the heteronuclear diatomic molecule, we employ the following external potential:

$$
v_{\mathrm{ext}}^{\mathrm{HM}}(x)=-\frac{1-\delta}{\sqrt{\left(x-\frac{R}{2}\right)^{2}+\sigma^{2}}}-\frac{1+\delta}{\sqrt{\left(x+\frac{R}{2}\right)^{2}+\sigma^{2}}},
$$

where $R$ is the distance of the hydrogen atoms and $\delta$ is the charge imbalance between the two nuclei. In this work, we set $R$ to 5 a.u, and $\delta$ to 0.2 a.u.

Figure 5(a) shows the exact ground-state electron density of the one-dimensional heteronuclear diatomic molecule, obtained by numerically solving the two-dimensional Schrödinger equation with the conjugate gradient method. Reflecting the charge imbalance between two nuclei, the electron density $\rho(x)$ is not symmetric. Figure 5(b) shows the exact exchange-correlation potentials of the effectively interacting Kohn-Sham systems. The exchange-correlation potential of the noninteracting Kohn-Sham system (red solid line) approaches different values in the positive and negative $x$ regions. The energy difference of the asymptotic values reflects the difference of the ionization potentials of the two

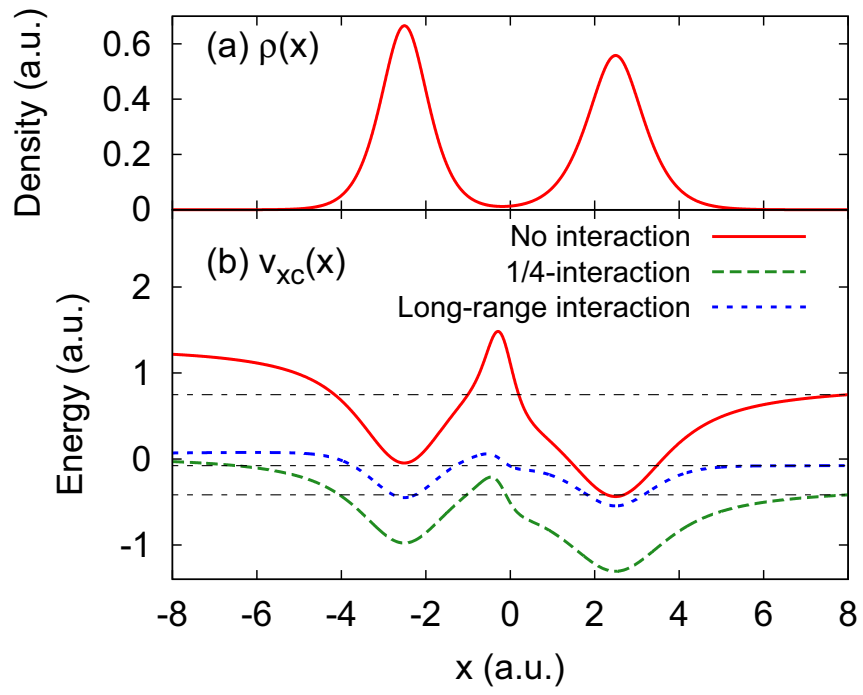

FIG. 5. (a) The exact ground-state density $\rho(x)$ of the onedimensional heteronuclear diatomic molecule. (b) The exact exchange-correlation potentials for the effectively interacting KohnSham systems. The results for the noninteracting system (red solid line), the 1/4-interacting system (green dashed line), and the longrange interacting system (blue dotted line) are shown. The horizontal black dotted lines show values of each potential $v_{\mathrm{xc}}(x)$ evaluated at $x=8$ a.u.

atoms. This feature is known as a step of the exact exchangecorrelation potential in heteroatomic molecules [17].

Note that, far from the molecule, the exact exchangecorrelation potential approaches the same asymptotic value on both the left and the right sides [18].

As seen from Fig. 5(b), the exchange-correlation potentials of the 1/4-interacting Kohn-Sham system (green dashed line) and those of the long-range interacting Kohn-Sham system (blue dotted line) show the a step feature weaker than that of the noninteracting system (red solid line). To quantify the size of the step feature, we evaluate the difference of the potential at $x=-8$ and 8 a.u., $\Delta_{s}=v_{\mathrm{xc}}(x=-8$ a.u. $)-v_{\mathrm{xc}}(x=$ 8 a.u.). The step size of the noninteracting Kohn-Sham system is $\Delta_{s}=0.47$ a.u., and that of the $1 / 4$-interacting Kohn-Sham system is $\Delta_{s}=0.39$ a.u. The long-range interacting KohnSham system provides the smallest step size, $\Delta_{s}=0.15$ a.u. Thus, one can clearly conclude that the effectively interacting Kohn-Sham systems significantly reduce the step feature of the exchange-correlation potential by transferring a part of the electronic correlation from the exchange-correlation potential to the explicit correlation in the reference wave function. Consistent with the above findings, the long-range interacting Kohn-Sham system has the largest reduction of the complex feature of the exchange-correlation potential. Thus, effective interaction based on the range separation is suggested to be a key to achieving an efficient description of static and dynamical correlation with the combined theory of DFT and wave-function theory.

\section{SUMMARY AND OUTLOOK}

In order to explore a possibility to combine DFT and wave-function theory, we investigated a mapping from a fully 
interacting problem to an effectively interacting problem instead of the conventional mapping to the noninteracting KohnSham system. To elucidate such mapping, we considered three kinds of effectively interacting Kohn-Sham systems. One is the usual noninteracting Kohn-Sham system. The second one is the 1/4-interacting Kohn-Sham system, where the effective interaction is set to the quarter of the full interaction. The last one is the long-range interacting Kohn-Sham system, where the short-range part of the full interaction is ignored.

To practically investigate the properties of the effectively interacting Kohn-Sham system, we first investigated the exact exchange-correlation potentials of the one-dimensional helium atom. As a result, we found that the asymptotic behavior of the exchange-correlation potential is determined by that of the residual interaction, which is defined as the difference of the full and the effective interactions. This fact further indicates a possibility to construct a good local density approximation for an effectively interacting Kohn-Sham system by optimally choosing a short-range residual interaction because the exchange-correlation potential vanishes as density vanishes and the nonlocal density dependence is expected be suppressed.

Next, we evaluated the occupation distribution of the natural orbitals of the ground-state wave function of the effectively interacting Kohn-Sham systems. As a result, we found that the occupations of the higher natural orbitals are significantly suppressed in the effectively interacting Kohn-Sham systems, especially in the long-range interacting Kohn-Sham system. This fact indicates that the effectively interacting Kohn-Sham systems offer an efficient decomposition of the electronic correlation into the dynamical correlation in the DFT part and the static correlation in the wave-function part.

Then, we investigated the exact exchange-correlation potentials of the one-dimensional hydrogen molecule. Consistently with the previous works $[17,18,47,48]$, we observed the spiky feature of the exchange-correlation potential. Once the effective interaction is turned on, the spiky feature is strongly suppressed. Furthermore, in the long-range interacting KohnSham system, the spiky feature completely vanishes and the exchange-correlation potential becomes smooth at the center of the molecule.

Finally, we investigated the exact exchange-correlation potentials of the one-dimensional heteronuclear diatomic molecule in order to study the step feature of the exchangecorrelation potential, which reflects the different ionization potentials of the two atoms [17]. Consistently with the above analysis, we found that the difficult step feature is significantly reduced in the effectively interacting Kohn-Sham systems, compared with the noninteracting Kohn-Sham system. Especially, the long-range interacting Kohn-Sham system shows the smallest step feature, indicating the effectiveness of the concept of the range separation in the effectively interacting Kohn-Sham systems.

Based on the above findings, we can conclude that the effectively interacting Kohn-Sham approach can open a way to efficiently describe the electronic correlation effect by the combination of DFT and wave-function theory, decomposing the electronic correlation effect into the DFT part and the wave-function theory part.

Another important fact is that the interacting Kohn-Sham scheme can be reduced to the hybrid functional, applying the Hartree-Fock approximation to the interacting KohnSham equation, Eq. (3). Therefore, the interacting Kohn-Sham scheme offers a possibility to improve the hybrid functional approximation within the formal theoretical framework of DFT, by adding the explicit correlation in the many-body Kohn-Sham wave functions.

In this work, we limited ourselves to the theoretical analysis of one-dimensional two-electron systems in order to investigate the exact properties of the interacting KohnSham systems. However, importantly, the above findings can be straightforwardly extended to multidimensional manyelectron systems and open a possibility to construct robust and efficient density functionals. For example, based on the weak nonlocality of the exchange-correlation potential of the interacting Kohn-Sham system, an accurate LDA may be constructed from the homogeneous electron gas with a suitable effective interaction. Furthermore, inspired by the $G W$ method and the screened hybrid-functional approach, a screening effect can be incorporated in an effective interaction of the Kohn-Sham system, and an accurate description of solid-state materials may be realized. These extensions of the present work to multidimensional many-electron systems are under way.

\section{ACKNOWLEDGMENTS}

This work was supported by the European Research Council (Grant No. ERC-2015-AdG694097), the Cluster of Excellence "Advanced Imaging of Matter" (AIM), and JST-CREST under Grant No. JP-MJCR16N5. Support by the Flatiron Institute, a division of the Simons Foundation, is acknowledged. S.A.S. gratefully acknowledges a fellowship from the Alexander von Humboldt Foundation.
[1] P. Hohenberg and W. Kohn, Phys. Rev. 136, B864 (1964).

[2] W. Kohn and L. J. Sham, Phys. Rev. 140, A1133 (1965).

[3] J. P. Perdew and A. Zunger, Phys. Rev. B 23, 5048 (1981).

[4] J. P. Perdew and Y. Wang, Phys. Rev. B 45, 13244 (1992).

[5] R. van Leeuwen and E. J. Baerends, Phys. Rev. A 49, 2421 (1994).

[6] J. P. Perdew, K. Burke, and M. Ernzerhof, Phys. Rev. Lett. 77, 3865 (1996).
[7] J. Tao, J. P. Perdew, V. N. Staroverov, and G. E. Scuseria, Phys. Rev. Lett. 91, 146401 (2003).

[8] T. Van Voorhis and G. E. Scuseria, J. Chem. Phys. 109, 400 (1998).

[9] J. Sun, A. Ruzsinszky, and J. P. Perdew, Phys. Rev. Lett. 115, 036402 (2015).

[10] A. D. Becke, J. Chem. Phys. 98, 5648 (1993).

[11] J. P. Perdew, M. Ernzerhof, and K. Burke, J. Chem. Phys. 105, 9982 (1996). 
[12] J. Heyd, G. E. Scuseria, and M. Ernzerhof, J. Chem. Phys. 118, 8207 (2003).

[13] M. Levy, J. P. Perdew, and V. Sahni, Phys. Rev. A 30, 2745 (1984).

[14] C. O. Almbladh and A. C. Pedroza, Phys. Rev. A 29, 2322 (1984).

[15] M. A. Buijse, E. J. Baerends, and J. G. Snijders, Phys. Rev. A 40, 4190 (1989).

[16] O. V. Gritsenko and E. J. Baerends, Theor. Chem. Acc. 96, 44 (1997).

[17] N. Helbig, I. V. Tokatly, and A. Rubio, J. Chem. Phys. 131, 224105 (2009).

[18] M. J. P. Hodgson, J. D. Ramsden, and R. W. Godby, Phys. Rev. B 93, 155146 (2016).

[19] K. Burke, Time-Dependent Density Functional Theory, Lecture Notes in Physics, Vol. 706 (Springer, New York, 2006), p. 181.

[20] J. P. Perdew and K. Schmidt, AIP Conf. Proc. 577, 1 (2001).

[21] M. G. Medvedev, I. S. Bushmarinov, J. Sun, J. P. Perdew, and K. A. Lyssenko, Science 355, 49 (2017).

[22] A. Szabo and N. S. Ostlund, Modern Quantum Chemistry (McGraw-Hill, New York, 1989).

[23] F. Aryasetiawan and O. Gunnarsson, Rep. Prog. Phys. 61, 237 (1998).

[24] W. G. Aulbur, L. Jönsson, and J. W. Wilkins, Quasiparticle Calculations in Solids (Academic, San Diego, 2000), pp. 1-218.

[25] G. Onida, L. Reining, and A. Rubio, Rev. Mod. Phys. 74, 601 (2002).

[26] M. J. van Setten, F. Weigend, and F. Evers, J. Chem. Theory Comput. 9, 232 (2013).

[27] W. M. C. Foulkes, L. Mitas, R. J. Needs, and G. Rajagopal, Rev. Mod. Phys. 73, 33 (2001).

[28] L. Stella, C. Attaccalite, S. Sorella, and A. Rubio, Phys. Rev. B 84, 245117 (2011).
[29] R. J. Hunt, M. Szyniszewski, G. I. Prayogo, R. Maezono, and N. D. Drummond, Phys. Rev. B 98, 075122 (2018).

[30] G. Mazzola, R. Helled, and S. Sorella, Phys. Rev. Lett. 120, 025701 (2018).

[31] O. V. Gritsenko, P. R. T. Schipper, and E. J. Baerends, J. Chem. Phys. 107, 5007 (1997).

[32] A. J. Cohen, P. Mori-Sánchez, and W. Yang, Science 321, 792 (2008).

[33] P. Mori-Sánchez, A. J. Cohen, and W. Yang, Phys. Rev. Lett. 102, 066403 (2009).

[34] D. H. Ess, E. R. Johnson, X. Hu, and W. Yang, J. Phys. Chem. A 115, 76 (2011).

[35] A. Seidl, A. Görling, P. Vogl, J. A. Majewski, and M. Levy, Phys. Rev. B 53, 3764 (1996).

[36] S. Grimme, Chem. Phys. Lett. 259, 128 (1996).

[37] P. Borowski, K. D. Jordan, J. Nichols, and P. Nachtigall, Theor. Chem. Acc. 99, 135 (1998).

[38] S. Grimme and M. Waletzke, J. Chem. Phys. 111, 5645 (1999).

[39] J. Gräfenstein, E. Kraka, and D. Cremer, Chem. Phys. Lett. 288, 593 (1998).

[40] M. Filatov and S. Shaik, Chem. Phys. Lett. 288, 689 (1998).

[41] M. Roemelt, D. Maganas, S. DeBeer, and F. Neese, J. Chem. Phys. 138, 204101 (2013).

[42] E. Fromager, J. Toulouse, and H. J. A. Jensen, J. Chem. Phys. 126, 074111 (2007).

[43] M. Levy and F. Zahariev, Phys. Rev. Lett. 113, 113002 (2014).

[44] Y. Wang and R. G. Parr, Phys. Rev. A 47, R1591 (1993).

[45] S. E. B. Nielsen, M. Ruggenthaler, and R. van Leeuwen, Eur. Phys. J. B 91, 235 (2018).

[46] P.-O. Löwdin, Phys. Rev. 97, 1474 (1955).

[47] O. V. Gritsenko and E. J. Baerends, Phys. Rev. A 54, 1957 (1996).

[48] M. J. P. Hodgson, J. D. Ramsden, T. R. Durrant, and R. W. Godby, Phys. Rev. B 90, 241107(R) (2014). 